\title{
Mechanism of transition to turbulence in a circular cylinder wake in a channel
}

\author{
Valery Molochnikov ${ }^{1, *}$, Alexander Mazo $^{2}$, Dmitriy Okhotnikov ${ }^{2}$ and Anna Goltsman ${ }^{1}$ \\ ${ }^{1}$ Kazan Scientific Center of RAS, Kazan, 420111, Russia \\ ${ }^{2}$ Kazan Federal University, Kazan, 420008, Russia
}

\begin{abstract}
Transition to turbulence in the circular cylinder wake has been studied experimentally and numerically at growing Reynolds number. Good agreement of calculation results with the flow visualization and measurements of instantaneous vector fields of velocity and vorticity has been demonstrated. The growing Reynolds number is shown to make large-scale vortex generation onset move upstream. It also triggers the transition to 3D flow pattern in the cylinder wake. This process is accompanied by non-monotonous behavior of the profiles of velocity and its turbulent fluctuations at equal distances from the cylinder. Nonmonotonous behavior of the cylinder drag has been revealed for the Reynolds numbers ranging from 120 to 300 .
\end{abstract}

\section{Introduction}

The regular Karman street onset in the wake of a cylinder in a cross-flow is attributed (depending on the literature source) to the range of Reynolds numbers $\mathrm{Re}=40-100$. Growing Re leads to the increase of the dimensionless shedding frequency (Strouhal number, $\mathrm{St}$ ), the latter being almost constant in the range of $\mathrm{Re} \approx 10^{3}-2 \times 10^{5}$. The curve $\mathrm{St}(\mathrm{Re})$ has been obtained for the first time by A. Roshko [1]. He showed that the separated shear layer and vortices formed downstream of the cylinder remained laminar in the range of $\operatorname{Re}=40-150$. Moving downstream, vortices dissipated due to viscous diffusion. At $150<\operatorname{Re}<300$, flow velocity fluctuations were observed in the cylinder wake. They were attributed to the laminar-turbulent transition in the separated shear layer. In this range of $\mathrm{Re}$, there is a non-monotonous dependence of the shedding frequency on the Reynolds number [1]. Authors [2] identified two scenarios of transition to turbulence, each of them in the certain Reynolds number range. Laminar turbulent transition at $200<\mathrm{Re}<400$ occurred inside the vortices and resulted from the deformation due to large-scale 3D effects. Growing Reynolds number made the transition onset move upstream; at $\operatorname{Re}>400$ the transition took place in the separated shear layer and resulted from the instabilities of Tollmien-Schlichting waves.

Late laminar-turbulent transition in the cylinder wake has been studied more thoroughly. Williamson [3] showed that the transition to turbulence in the wake had two stages. The first one was observed at $\mathrm{Re}=170-180$ and was characterized by the onset of

*Corresponding author: vmolochnikov@,mail.ru 
vortex loops and their hysteresis. The second one occurred at $\mathrm{Re}=230-260$ and corresponded to the changes in small-scale spanwise vortex pattern. Both stages were accompanied by $\mathrm{St}(\mathrm{Re})$ discontinuities. The latter were mentioned also in [4] where the transition to turbulence in the cylinder wake at $\mathrm{Re}=100-300$ was studied by numerical solution of 3D Navier-Stokes equations. Wavelike shape of large-scale vortices in spanwise direction at $\mathrm{Re}=220$ was also discovered in [4]. Authors [5] analyzed experimental data and numerical simulation results and established four physically different instabilities in the cylinder wake in the range of $\operatorname{Re}=160-230$. One of them was called "vortex-adhesion mode" with Karman vortices that seemed to adhere to a steady or slowly moving point on the cylinder for many periods [5]. The other three instabilities had different wavelengths of $3 \mathrm{D}$ global modes. A number of authors note that flow turbulization around the cylinder is accompanied by the transition to $3 \mathrm{D}$ wake structure [6].

All available research results dealing with the laminar-turbulent transition in the circular cylinder wake have been obtained for the case of almost unbounded external flow. Besides, the cylinder was always located symmetrically relative to the channel walls. There are no data on the features of transition to turbulence in the cylinder wake in the case when the cylinder is located near the wall. There are only scarce data available on the side wall effect on these processes. However, it is well known that spiral motion of fluid from the side walls of the channel towards its symmetry plane is formed downstream of a semicylindrical rib mounted on the channel wall. This motion governs the processes of laminar-turbulent transition behind an obstacle and formation of large-scale vortices in the mixing layer. There are no data available on existence of such motion past a circular cylinder near the channel wall.

The present work elaborates on experimental results and DNS of the flow structure in the cylinder wake in a cross-flow when the cylinder is located near the rectangular channel wall and the Reynolds number range is $\mathrm{Re}_{\mathrm{d}}=125-250$. The flow pattern has been described together with its evolution for the case of transition to turbulence in the cylinder wake.

\section{Experimental procedure}

The experiments were carried out in a rectangular test section with the width $B=50 \mathrm{~mm}$, height $H=20 \mathrm{~mm}$ and length $L=250 \mathrm{~mm}$ (fig. 1). The air was pumped through the test section by a vacuum pump. A circular cylinder with the diameter $d=3 \mathrm{~mm}$ was mounted across the test section parallel to its wide wall at the distance of $l=100 \mathrm{~mm}$ from the channel inlet. The distance between the cylinder axis and the nearest channel wall was equal to the cylinder diameter, $d$. Reynolds numbers based on the average velocity in the channel, $U_{0}$, and the cylinder diameter were $\operatorname{Re}_{\mathrm{d}}=U_{0} d / v=135,167,233$, and 276. The experiments included flow visualization and measurements of dynamics of instantaneous velocity vector fields in the cylinder wake. Smoke-wire visualization technique was used for flow visualization. The videos were recorded by a high-speed monochrome camera Fastec HiSpec in a light sheet in $x 0 y$ plane (channel symmetry plane) and in the plane parallel to $x 0 z$ at $y=4.5 \mathrm{~mm}$ (tangent to the cylinder generatrix). Instantaneous velocity vector fields in the cylinder wake were measured by SIV technique [6].

DNS was performed on the basis of Navier-Stokes equations for incompressible fluid. The computational domain geometry matched the test section studied in experiments. Calculations were performed in ANSYS Fluent 15.0 software using the second-order finite volume method, and the computational grid was built using locally-structured approach [7]: the domain was filled with a set of structured grid segments, which were connected by nonstructured insertions. The grid contained $10^{6}$ cells and was refined near the solid walls and in the wake. Minimal dimensionless grid spacing normal to the cylinder generatrix was 0.02 
(all dimensions were normalized by the cylinder diameter). The cell size in the middle wake was 0.15 . Minimal grid spacing normal to the wall in the boundary layer of the test section was 0.01 . The time step was 0.05 . Weightless particles were visualized to imitate the smoke wire used in the experiments.

\section{Results and discussion}

Good agreement between the calculations, flow visualization and SIV measurements has been established (fig. 1). Main regularities in laminar-turbulent transition in the cylinder wake have been obtained. A closed laminar separation region has been discovered immediately downstream of the cylinder at $\mathrm{Re} \leq 167$. It consists of two steady symmetric vortices and is followed by a regular large-scale vortex street further downstream. Wake flow remains quasi two-dimensional across almost the whole channel span except for the boundary layer on the channel side walls. The wake flow is laminar: second invariant of the Reynolds stress tensor $\mathrm{II}_{\mathrm{T}}=\sqrt{\tau_{i j} \tau_{j i}}$ remains zero throughout the whole wake region, and generated vortices maintain their integrity across the channel span.
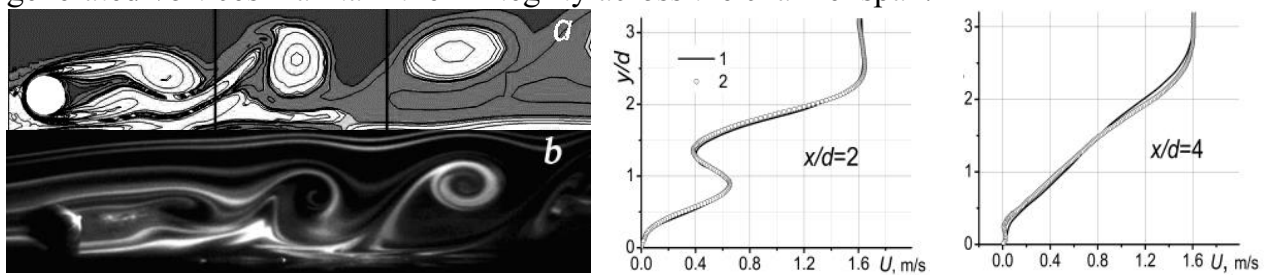

Fig. 1. Velocity field downstream of the cylinder and velocity profiles in the wake at $\operatorname{Re}=235$ : $a$-calculation; $b$ - flow visualization; 1 - calculation; 2 - SIV measurements

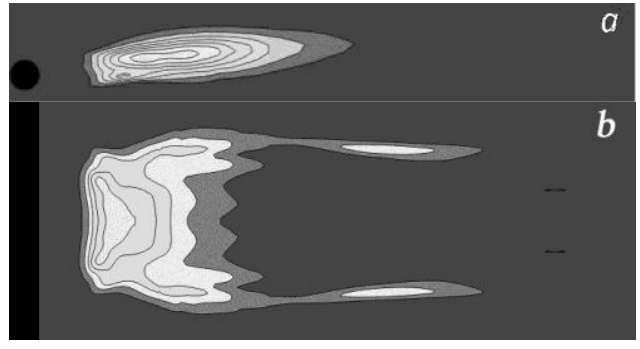

Fig. 2. Second invariant of the Reynolds stress tensor in the cylinder wake at $\operatorname{Re}=233: a-$ vertical plane; $b$-horizontal plane

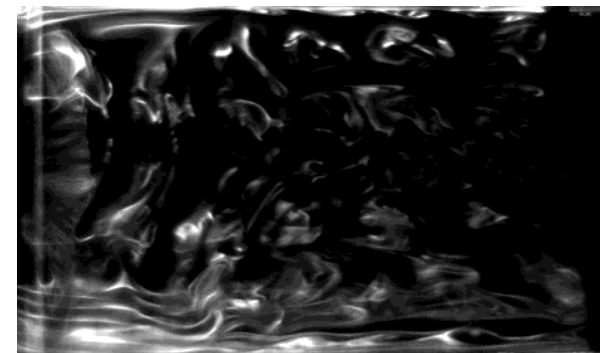

Fig. 3. Cylinder wake visualization at $\mathrm{Re}=233$ (horizontal plane)

The region of vortex generation onset shifts upstream with the growing Reynolds number. Reynolds stresses are non-zero already at $\mathrm{Re}=233$ (fig. 2), and the features of 3D flow are observed in the cylinder wake (fig. 3): while moving downstream, vortices split into several vortex clots. However, the near-wake flow (where there are still no Karman vortices) remains laminar.

Further increase of the Reynolds number leads to the filling of high-frequency part of the velocity fluctuation spectrum, which indicates the developed turbulent flow onset. The spanwise profile of velocity is almost uniform at $\mathrm{Re}=135$ (fig. 4,a) but has several local extrema already at $\mathrm{Re}=276$ (fig. 4, $b$ ). Turbulent disturbances are localized in the middle wake of the cylinder; spanwise maxima in Reynolds stress are in the wake region. 

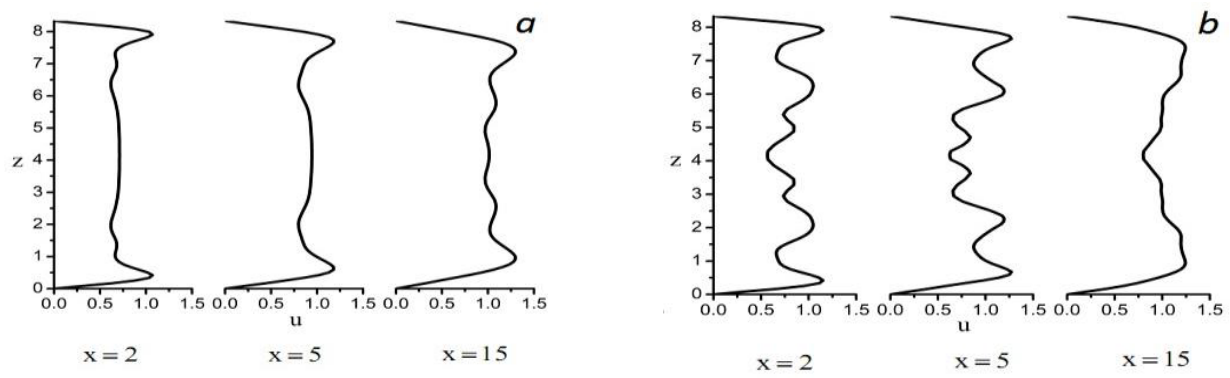

Fig. 4. Spanwise profiles of flow velocity in the cylinder wake: $a-\operatorname{Re}=135 ; b-276$

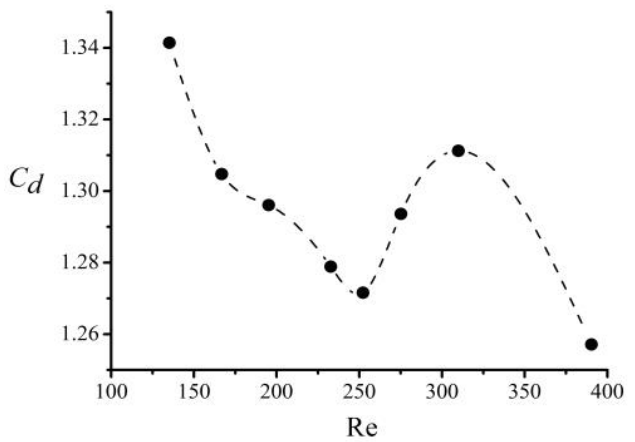

Fig.5. Cylinder drag coefficient vs Re

Thus, the transition to turbulence in the cylinder wake results from the flow instability in the separated shear layer at some distance from the cylinder. Nonmonotonous behavior of the cylinder drag coefficient as a function of the Reynolds number has been revealed in the range of $\mathrm{Re}=120-300$ (fig. 5). The latter corresponds to transition to turbulence in the cylinder wake. In the whole considered Reynolds number range there is no spiral motion of fluid from the side walls to the channel symmetry plane.

\section{Conclusions}

An experimental study and DNS of the pulsating cross-flow past a circular cylinder mounted near the channel wall have been performed in the range of the Reynolds number $\mathrm{Re}=135-276$. Good agreement between the experimental and simulation data has been obtained. It has been established that a street of regular large vortices is formed downstream of the cylinder surface that is the most distant from the channel wall. Features of 3D flow behavior have been observed in the wake starting from $R e \approx 230$. The transition to turbulence downstream of the cylinder has been shown to result from the shear layer instability at some distance from the cylinder. Non-monotonous behavior of the cylinder drag has been revealed in the range of $\operatorname{Re}=120-300$.

This study was supported by the Russian Science Foundation (Project no.16-19-10336).

\section{References}

1. A. Roshko, NACA Report 1191 (1958)

2. F.M. S. Bloor, J. Fluid Mech. 19, 290 (1964)

3. C.H.K. Williamson, Phys. Fluids. 31, 3165 (1988)

4. H. Persillon, M. Braza, J. Fluid Mech. 365, 23 (1998)

5. H.-Q. Zhang, U. Fey, B. R. Noack, M. Konig, H. Eckelmann, Phys. Fluids 7, 779 (1995)

6. N.I. Mikheev, N. S. Dushin, Instr. Exp. Techn. 59, 882 (2016)

7. A.B. Mazo, D.I. Okhotnikov, Lobachevskii J. Math. 37, 360 (2016) 